\title{
Las redes sociales: medio facilitador en el desarrollo del marketing de causa
}

Sandra Yanet Velazco Flórez*, Alexandra Abuchar Porras**, Orlando García Hurtado***

\section{Resumen}

Muchos negocios o comercios viven obsesionados con las cifras, sin discurrir en que, por cada labor destinada a la visibilización, podrían, además, coadyuvar significativamente a otras causas como medio hacia el bienestar común. Este escrito aborda el marketing de causa. Este busca, con sus acciones de visibilidad de marca y de comercio, involucrarse en cambios significativos y emocionales de una gran parte de los consumidores. Con el marketing de causa se persiguen objetivos claros, como los de aumentar las propias ventas y contribuir con las metas de algunos comercios no lucrativos. Se busca además mostrar cómo el comercio electrónico, o e-commerce, se ha combinado con este tipo de marketing, lo que le da valor al papel de las redes sociales para potenciar un producto o servicio con mayor influencia emocional y sentido social, y así procurar una mayor compenetración de las personas tanto con la marca como con el medio ambiente. Sin duda alguna, el marketing de causa, junto con las redes sociales, se están convirtiendo en un gran desafío y beneficio de competitividad para muchos negocios que han tomado la decisión de aplicarlo dentro de sus estrategias de comunicación, promoción y difusión de su marca o producto.

Palabras clave: comercio electrónico, marketing, marketing con causa, redes sociales

\begin{abstract}
Many businesses and shops point their effort to figures without realizing that for each task that is carried out, they can also significantly contribute to causes for the common good. This paper addresses the cause marketing, which seeks, with its actions of brand and trade visibility, the engagement in significant and emotional changes of a large part of consumers with clear objectives, such as increasing their own sales and contribute to the goals of some nonprofit businesses. In addition, it is shown how the Electronic Commerce, or e-commerce, has been added to this type of marketing, attaching to the discussed topic the role of social networks seeking to enhance a product or service with greater emotional influence and social sense, as well as a greater understanding of people with both the brand and the environment. Without doubts, cause marketing, along with social networks, is becoming a great challenge and benefit of competitiveness for many businesses, which have decided to apply it within their communication strategies, promotion and dissemination of their brand or product.
\end{abstract}

Keywords: Cause Marketing, e-commerce, Marketing, Social Media

* Docente Universidad Distrital Francisco José de Caldas. Contacto: sandrayvelazcof@correo.udistrital.edu.co

** Docente Universidad Distrital Francisco José de Caldas. Contacto: aabucharp@udistrital.edu.co9

*** Docente Universidad Distrital Francisco José de Caldas. Contacto: ogarciah@udistrital.edu.co 


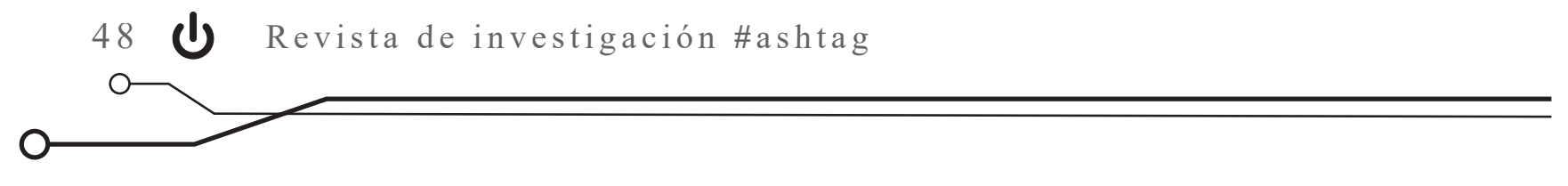

\section{Introducción}

Al hablar de comercio electrónico o e-commerce es común que se cree en la mente de muchas personas una imagen relacionada con la compra y venta de ciertos productos vía online, y que la atención se dirija al aspecto mercantil, es decir, pensar que la retribución económica en este tipo de actividad es lo más relevante. Sin embargo, existe una amplia parte del comercio electrónico menos "codicioso", que viene implementando acciones inmersas en el denominado marketing

\section{El comercio electrónico}

En la actualidad, el comercio electrónico es una herramienta necesaria a fin de seguir logrando y manteniendo los niveles de competitividad requeridos en el mercado. Aunque con ciertas limitaciones, el desarrollo del e-commerce puede ser cuantificable y razonablemente predecible (Gariboldi, 1999).

Como muchos otros términos, existe una gran diversidad de definiciones establecidas con respecto al concepto de comercio electrónico, tanto por organismos internacionales como por profesionales relacionados con el tema. En tal sentido, y de acuerdo con la Organización Mundial del Comercio, OMc, el comercio electrónico es "la distribución, mercadeo, venta o entrega de social, y que actualmente se conoce como marketing de causas sociales.

En este artículo se dará una visión global del marketing de causa, su accionar mediante el uso y disposición de las redes sociales, para mostrar su potencial aporte, no solamente a un producto, una marca o un comercio, sino también ver cómo genera un servicio de competitividad y alto sentido social y emocional.

bienes y/o servicios hecha con medios electrónicos" (OMC, 2016).

Así mismo, existen diversas relaciones electrónicas entre diferentes actores, tales como negocios, consumidores y gobiernos, lo que produce valiosos beneficios, especialmente en el funcionamiento transaccional del comercio entre negocios y consumidor final (véase figura 1). De esa manera, se han desarrollado diferentes segmentos de comercio electrónico, dentro de las cuales se destacan el tipo B2B (comercio que se realiza entre negocio y negocio), el B2C (comercio que se realiza entre negocio y consumidor), C2C (comercio que se realiza entre consumidores únicamente) y el B2G (comercio que se da entre consumidores y el gobierno) (CRC, 2017). 
Figura 1. Beneficios del Comercio Electrónico en las empresas y los consumidores
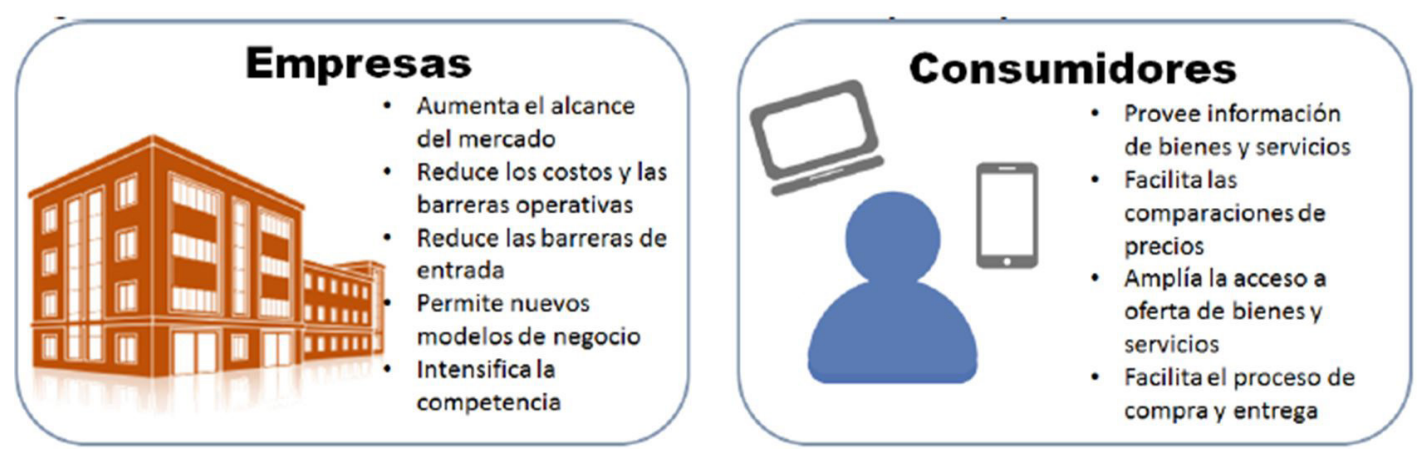

Fuente: CRC, 2017

Si bien el segmento B2B representa una mayor proporción del e-commerce, las actividades B2C muestran un rápido crecimiento. Por ejemplo, en los Estados Unidos, su proporción en el comercio electrónico aumentó del 2,6 \% al 4,5 \% entre 2002 y 2012. En la Federación Rusa, las transacciones $\mathrm{B} 2 \mathrm{C}$ aumentaron rápidamente, casi tres veces más que las B2B en 2013 (iKS Consulting, 2013; Organización de las Naciones Unidas, ONU, 2015).
Ahora bien, la evolución del internet ha potenciado enormemente el intercambio de información, al hacerlo de forma más rápida y eficiente, lo que posibilita un acceso fácil y amplio a bienes y servicios. Internet aumenta constantemente y su nivel de penetración mundial, a febrero de 2017, ha sido del 49.6 \% de la población mundial, según indicadores del sitio Internet World Stats (Islas, 2017).

Figura 2. Nivel de penetración global del Internet a enero de 2017.

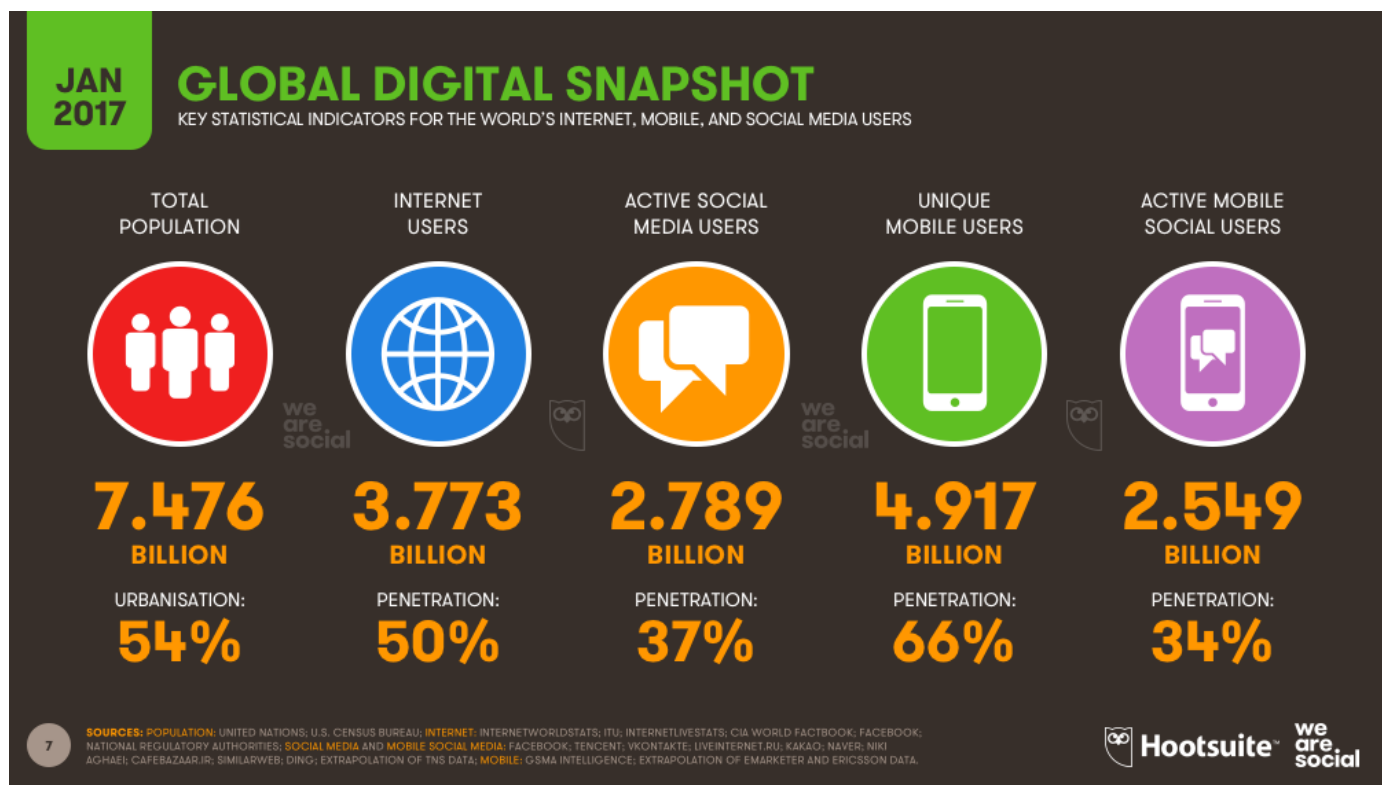




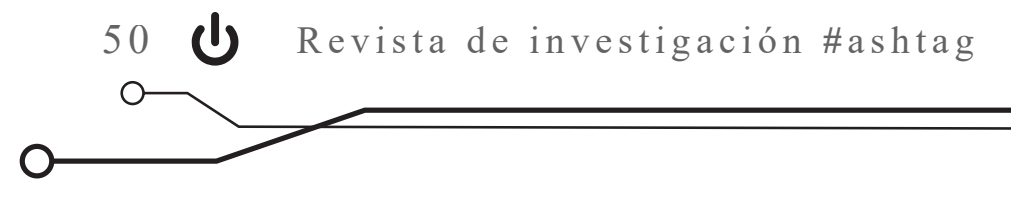

Por otro lado, la masificación de los teléfonos inteligentes ha acelerado el crecimiento del B2C, lo que ha facilitado las opciones de hacer transacciones y compras en línea, el escaneo de códigos, búsqueda de productos, así como la posibilidad de compartirlos en redes sociales (CRC, 2017). Finalmente, gracias al incremento en el uso de las redes sociales, se prevé la entrada del comercio basado en las redes sociales o social commerce (Ahmed, 2015). Una muestra de ello son los anuncios de Facebook, Pinterest y Twitter, en tanto que agregan botones para la compra de productos y servicios ofrecidos por los usuarios (Hughes, 2015).

El desarrollo tecnológico ha marcado un cambio organizacional significativo en el sector empresarial a nivel global al potenciar su participación en la era digital, mediante las posibilidades que ofrece el internet y las Tic en el mundo de los negocios. También reduce los costos de entrega de productos y servicios, elimina las limitaciones geográficas, lo que facilita el intercambio entre consumidores y vendedores (Barrientos, 2017).

\section{El marketing digital y las redes sociales}

Las empresas han sobrellevado muchos cambios en muy poco tiempo desde principios del siglo XXI, por los permanentes avances tecnológicos. El uso de las TIC aumenta día a día en las empresas con el fin de dar a conocer sus productos y reconocer a sus clientes (Durmaz, 2016).

En este contexto, la tendencia global del marketing digital consiste en una combinación conjunta de diversas formas de marketing, es decir, la implementación combinada de técnicas del marketing tradicional junto con las técnicas de los medios actuales, lo que puede ser visto como un componente del e-commerce, por lo que puede contener la gestión de contenidos, la reputación en línea, las relaciones públicas, el servicio al cliente y las ventas (Salazar, 2017).

Dentro del marketing digital, internet se ha convertido en una poderosa e indispensable herramienta que ha logrado mejorar la forma en que una empresa hace sus negocios y no únicamente como un método de venta o publicidad adicional. Como elemento esencial, el marketing digital debe estar centrado en el consumidor y se debe procurar una interacción entre la campaña de publicidad, la promoción y el público que la recibe. En este sentido, las redes sociales han mostrado un importante crecimiento en inversión sobre los métodos de publicidad y promoción tradicionales, lo que pone de presente que las redes de amplia utilización incorporan y concentran metodologías para desarrollar publicidad efectiva en ellas (Salazar, 2017).

Las empresas que practican el comercio electrónico encuentran en las redes sociales un sitio que les hace saber qué quiere la gente, qué les disgusta y cómo poder repararlo. Tal y como dice Trey Pennington: "Las empresas que entienden las redes sociales son las que dicen con su mensaje: te veo, te escucho y me importas" (Pennington, 2011. Traducción propia).

Hoy en día la preocupación de las personas por las causas sociales es creciente y se ve reflejada en las redes sociales, en donde es vista y estudiada por las empresas, en especial por aquellas en el ámbito $e$-commerce. Muchas veces esta estrategia de hecho se aprovecha para ganar mayor 
aceptación del público y así mostrar un lado más solidario y amigable, "humanizándose", para crear una conexión con los clientes e incrementar su lealtad. Además, quienes hacen uso de dicha estrategia se comprometen a ofrecer parte de las ganancias de la venta a una causa solidaria, lo que incentiva la toma de decisión de compra con base en un argumento solidario (Altagracia, 2012).

\section{El marketing de causa como estrategia}

El marketing de causa es una herramienta estratégica que implica tres grandes actores: empresa, causa y consumidor (véase figura 3). Esta estrategia, en ocasiones confundida con el marketing social, busca impactar positivamente tanto en los consumidores como en los beneficiarios de la acción y las empresas (Kotler, 2011).

Figura 3. Agentes implicados en una acción de marketing de causa.

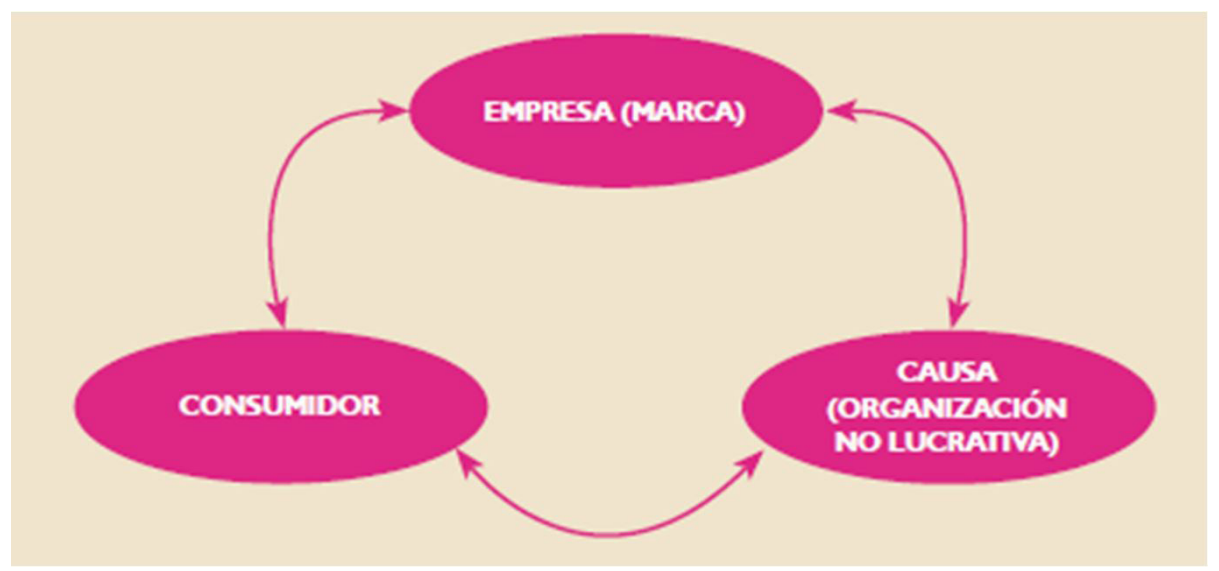

Fuente: Buil, 2012.

El marketing de causa se ha convertido en una de las principales iniciativas de responsabilidad social que llevan a cabo los comercios. Dentro de sus características más destacadas, se encuentran, primero, las que conllevan una colaboración entre un comercio lucrativo y uno no lucrativo, como apoyo de una causa social; y segundo, que el donativo del comercio o empresa está condicionado por las ventas consumadas. En otras palabras, depende del comportamiento del consumidor si adquiere o no el producto "solidario" (Galán, 2017). Algunos ejemplos de organizaciones sin ánimo de lucro y marcas exitosas a nivel global que aplican este tipo de estrategias se muestran a continuación.

McDonald's. Esta importante cadena de restaurantes cada año realiza una jornada solidaria denominada Mc Día Feliz, cuya finalidad es recaudar fondos para contribuir con la salud y bienestar de los niños, a través de la fundación Casa Ronald McDonald's. Los recursos obtenidos por la venta de su famoso producto Big Mac son destinados a la fundación en su totalidad. 


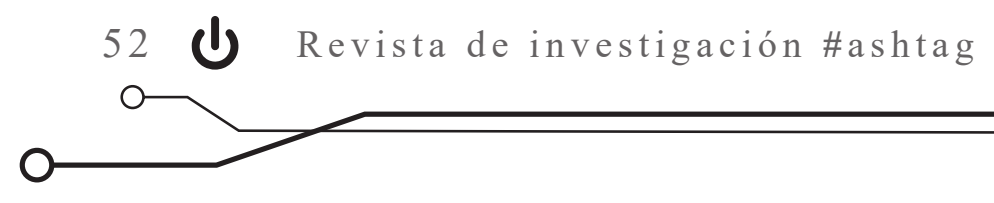

Esta campaña es la más grande realizada en América Latina y conmemora la Convención de los Derechos de los Niños, firmada en 1989 por la oNU y la Unicef (El Tiempo, 2011).

M4c. Esta es una plataforma tecnológica multidisciplinaria, que ayuda a obras sociales en pro de cambios integrales y desarrollo social. M4c enfoca su actuar en áreas de educación, formación, emprendimiento, y de proyectos que produzcan bienestar social a largo plazo. Con estas iniciativas se pueden crear plataformas de e-commerce para emprendedores sociales, organizaciones no gubernamentales y fundaciones con escasos medios tecnológicos para desarrollar un canal de ventas online. También buscan oportunidades para que todas las personas puedan desarrollarse de forma libre y puedan tener una vida digna (Padilla, 2015).

Ubergiving. En septiembre de 2016, la crisis de los refugiados europeos causó hechos lamentables, como la muerte de Alan Kurdi, de tres años de edad. Ante esta situación, la plataforma Uber decidió proporcionar asistencia logística permitiendo a las personas el dar y solicitar un servicio de automóvil para recoger donaciones, como mantas y ropa, que serían entregadas a socios benéficos locales como Save the Children, en el Reino Unido. Uber escaló esta iniciativa muy rápidamente: la implementó en 46 ciudades de Europa, en las que hubo grandes aportaciones. La compañía utilizó las redes sociales, los blogs, correos electrónicos y pantallas de presentación para comercializar UberGiving (Davis, 2016).
Pampers y la alianza de Unicef. Desde 2003 se han financiado 300 millones de vacunas contra el tétanos a través de la asociación Pampers y Unicef. El éxito de esta iniciativa de comercialización relacionada con causas a escala mundial radica en su mensaje simple, pero que salva vidas: "1 paquete $=1$ vacuna". Tal es su éxito que la docente Linda Scott, de la Universidad de Oxford, desarrolló un estudio de caso como un ejemplo de excelente práctica, en el que se evidencia que la marca de Unicef contribuyó sustancialmente al poder de la campaña para construir nuevos negocios para Pampers, al tiempo que logró nuevas fuentes de donaciones para Unicef a escala considerable (Institute of fundraising, 2017).

Small Business Day de American Express. American Express lanzó Small Business Saturday, como apoyo a las pequeñas empresas en EE. UU. en 2010. Para 2012, aproximadamente 73 millones de personas salieron a comprar en pequeñas empresas, y más de 1000 vecindarios se inscribieron para apoyar la iniciativa en 2013. Para 2014, se estimó que 14300 millones de dólares se estaban gastando en ese solo día. La clave del éxito de esta iniciativa se centró en que los participantes tenían que recibir recursos de diseño útiles para difundir el mensaje de la iniciativa. En este sentido, American Express diseñó piezas creativas como letreros, publicaciones sociales, mapas de búsqueda del tesoro, hojas de recetas y pasaportes temáticos para apoyar a sus Campeones del vecindario, hombres y mujeres que se comprometieron a celebrar formalmente el Sábado de Pequeños Negocios en sus áreas (Busche, 2015). 
Figura 4. Diseño de pasaportes temáticos para apoyar a la campaña Campeones del Vecindario de American Express 2014.

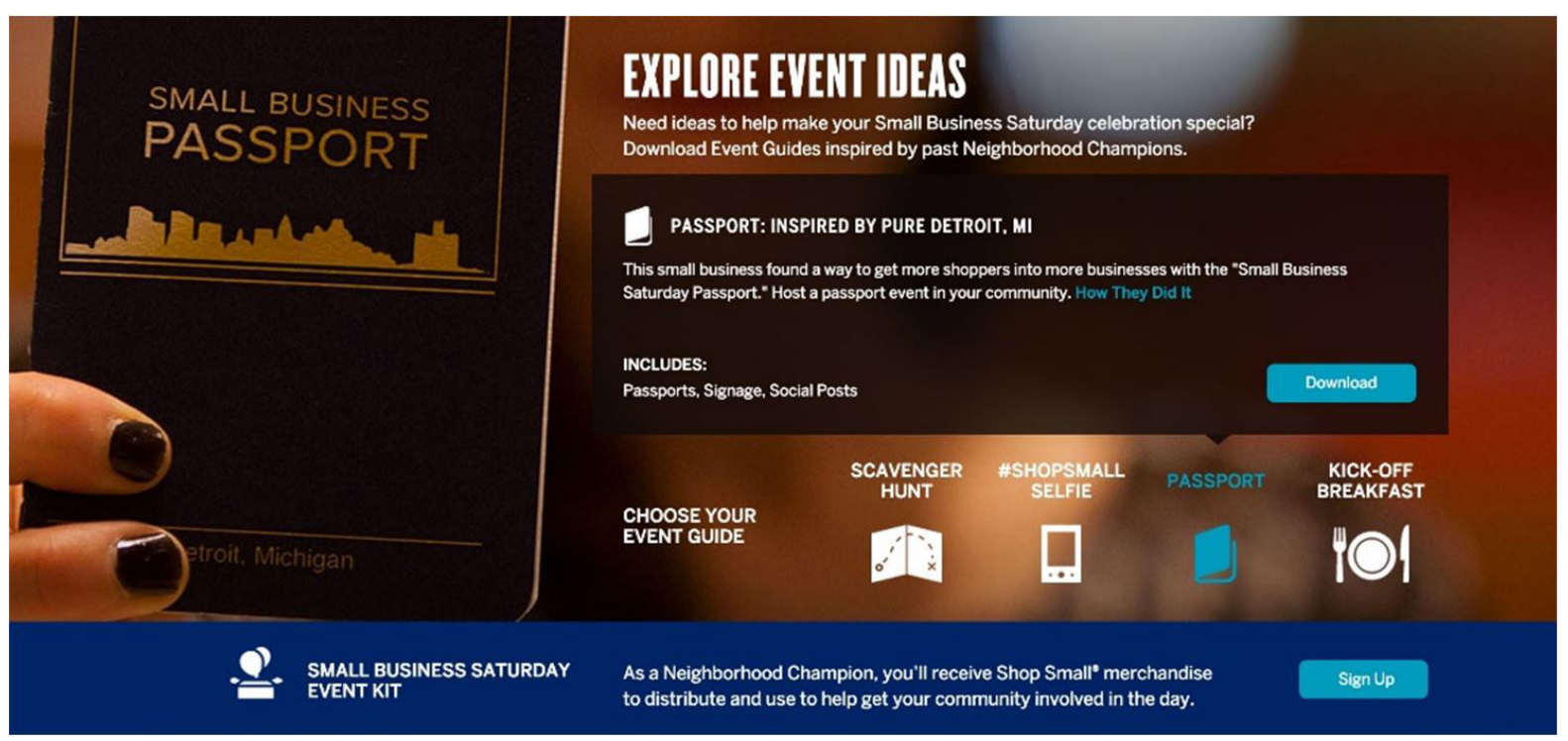

Fuente: Busche, 2015.

\section{Prospectiva}

Para que este tipo de iniciativas tenga éxito, se debe asumir una serie de retos, como el de llegar al público adecuado o a la mayor cantidad posible de consumidores. Por esta razón, el papel de las redes sociales es relevante para lograr una mayor cobertura, ya que a través de su uso es posible no solo convencer, sino también comunicar, promocionar y difundir información con mayor facilidad a los usuarios, teniendo en cuenta que el tiempo que ellos dedican a las redes sociales es cada vez mayor.

Con las redes sociales las personas no solo pueden compartir sus experiencias como clientes de marcas y/o productos. También pueden incluso exigir ciertos resultados o compromisos a marcas o empresas. En ese sentido, se hace relevante el hacer uso de la de la información sobre los usuarios, como sexo, edad, ubicación geográfica, etc., pues esta puede resultar de gran utilidad como guía para las empresas al momento de promocionarse y de la forma acertada de hacerlo, lo que deja en evidencia el grado de complicidad entre el comercio electrónico y las redes sociales.

El marketing social de causa se muestra como una iniciativa de buenas prácticas, pensada para aquellas empresas que buscan un interés más allá que el de lucrarse. Tal vez, como dice Leslie Bradshaw, directora de Jess3, "como en una relación, los usuarios valoran las marcas que den más valor del que piden". Generalmente, como consumidores nos apasionamos y nos dejamos llevar por los sentimientos, y bajo esta condición podríamos decir que quien nos genere emociones, tiene una alta probabilidad de poder persuadirnos de emprender algo y de llevarnos en un juego sano en relación con marketing de causa. 


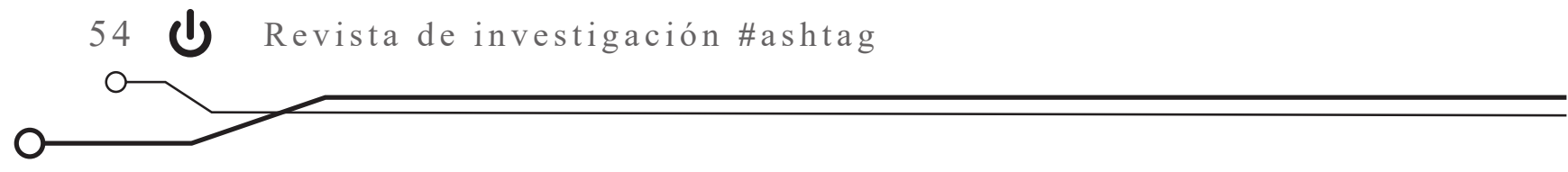

\section{Conclusiones}

En estos tiempos, el marketing de causa se ha transformado en una valiosa ventaja competitiva para los negocios que han querido tomar la apropiada decisión de implementar esta iniciativa como estrategia en sus diferentes actividades de promoción, comunicación y difusión de su marca o su producto.

Se puede considerar que el marketing de causa es un ejemplo de buenas prácticas que contribuyen sustancialmente al poder de las marcas, para promocionar, comunicar, divulgar y construir nuevos productos y negocios, a la vez que coadyuva con causas sociales, y ser un bonus para aquellos negocios pertenecientes al estilo e-commerce.

Aunque el concepto de marketing de causa no es reciente, sí lo es el impresionante desarrollo que ha tenido. Sin duda, mucho o todo se da gracias al impactante desarrollo y evolución de las nuevas tecnologías de la información y a la formidable repercusión que han provocado las redes sociales actualmente. Esto muestra que las campañas de marketing de causa, de hecho, pueden ir desde pequeños esfuerzos hasta las más grandes causas a nivel mundial.

\section{Referencias}

Ahmed, M. (mayo 28 de 2015). Is Social Media the Biggest Influencer of Buying Decisions? Social Media Today [entrada de blog]. Recuperado de: https:// bit.ly/333GYPO

Altagracia, L. (2012) Marketing Social [entrada de blog]. Recuperado de: https://bit.ly/3h9nFtg

Barrientos, P. (2017). Marketing + internet = e-commerce: oportunidades y desafíos. Revista Finanzas y Política Económica, 9(1), 41-56 ISSN 2248-6046.

Buil, I., Melero, I., y Montaner, T. (2012). La estrategia de marketing con causa: Factores determinantes de su éxito. Universia Business Review, (36), 90-107 ISSN: 1698-5117. Disponible en: https:/ / bit.ly/2Rahtac

Busche, L. (2015). Cause marketing: How these 20 visual campaigns created massive impact. Recuperado de: https://bit.ly/2R5zwOt

Comisión de Regulación de Comunicaciones-CRC. (2017). El Comercio Electrónico en Colombia. Análisis integral y perspectiva regulatoria. Recuperado de: https:// bit.ly/3m5MRop

Davis, B. (25 de octubre de 2016) Cause marketing: Examples from Uber, Starbucks \& JetBlue [entrada de blog]. Recuperado de: https:/ / bit.ly/3h5865X

Durmaz, Y. y Efendiaoglu, I. (2016). Travel from traditional marketing to digital marketing. Global Journal of Management and Business Research, 16(2), 34-40. 
El Tiempo (16 de noviembre de 2011) Este 18 de noviembre McDonald's celebrará el McDía Feliz. El Tiempo [en línea]. Recuperado de: https:// bit.ly/336aYuj

Galán, M. (2017). Marketing con causa: una mirada a Latinoamérica. El caso de Uruguay. Cuadernos de RSO, 5(2). 45-61.

Gariboldi, G. (1999) Comercio Electrónico: Conceptos y reflexiones básicas. Buenos Aires: Bid Intal. Institute for the Integration of Latin America and the Caribbean. ISBN 9507380787

Hughes, B. (2 de diciembre de 2015). Will 2016 Be the Year for Social Commerce? Small Business Trends [entrada de blog]. Recuperado de: https://bit.ly/2Fajkcp

iKS Consulting. (10 octubre de 2013). Retail E-Commerce Market in Russia. Year 2013 Preliminary Results [entrada de blog]. Recuperado de: https://bit.ly/35g9XTg

Institute of Fundraising. (2015). Great examples of cause related marketing. [entrada de blog]. Recuperado de: https:/ / bit.ly/2GG1BtM

Islas, O. (21 abril de 2017) Penetración mundial de Internet. El Universal. Recuperado de: https:/ / bit.ly/324w9hd

Kotler, P. (2011). Social Marketing: Influencing Behaviors for Good. 4a ed. Thousand oaks, California: Sage. ISBN: 9781412981491

Organización de las Naciones Unidas (onu) (2015). Informe sobre la economía de la información 2015 - Liberar el potencial del comercio electrónico para los países en desarrollo. Informe presentado en la Conferencia de las Naciones Unidas sobre Comercio y Desarrollo. Recuperado de: https:// bit.ly/3bJ7ty5

Organización mundial del Comercio (омC) (2016). Comercio electrónico: Programa de trabajo. Recuperado de: https:/ / bit.ly/3i9L4MC

Padilla, K. (19 agosto de 2015) Ejemplos de marketing social en el E-commerce. [entrada de blog]. Recuperado de: https:/ / bit.ly/3bGFxKW

Pennington, T. (2011). Rethinking marketing with Trey Pennington: the Copernican shift. Recuperado de: https:// bit.ly/2FmasQE

Salazar, A., Paucar-Coque, L. y Borja-Brazales, Y. (2017). El marketing digital y su influencia en la administración empresarial. Dominio de Las Ciencias, 3(4), 1161-1171. ISSN: 2477-8818

Wearesocial. (2017). Digital in 2017: Global overview. Recuperado de https:/ / wearesocial.com/ special-reports/digital-in-2017-global-overview 